\title{
The effects of ergot and non-ergot-derived dopamine agonists in an experimental mouse model of endometriosis
}

\author{
Francisco Delgado-Rosas, Raúl Gómez, Hortensia Ferrero, Francisco Gaytan¹, \\ Juan Garcia-Velasco, Carlos Simón and Antonio Pellicer \\ Fundacion IVI-Instituto Universitario IVI, INCLIVA, Universidad de Valencia, C/Guadassuar 1 Bajo, 46015 Valencia, \\ Spain and ${ }^{1}$ Department of Cell Biology, Physiology and Immunology, School of Medicine, University of Córdoba, \\ Cordoba, Spain \\ Correspondence should be addressed to A Pellicer; Email: antonio.pellicer@ivi.es
}

F Delgado-Rosas and R Gómez contributed equally to this work

\begin{abstract}
Implantation of a retrogradely shed endometrium during menstruation requires an adequate blood supply, which allows the growth of endometriotic lesions. This suggests that the development of endometriosis can be impaired by inhibiting angiogenesis. The growth of endometriotic foci is impaired by commercial oncological antiangiogenic drugs used to block vascular endothelial growth factor (VEGF) signaling. The dopamine agonist cabergoline (Cb2) inhibits the growth of established endometriosis lesions by exerting antiangiogenic effects through VEGFR2 inactivation. However, the use of ergot-derived $\mathrm{Cb2}$ is associated with an increased incidence of cardiac valve regurgitation. To evaluate the potential usage of non-ergot-derived dopamine agonists for the treatment of human endometriosis, we compared the efficacy of quinagolide with that of $\mathrm{Cb2}$ in preventing angiogenesis and vascularization in a heterologous mouse model of endometriosis. Nude mice whose peritoneum had been implanted with eutopic human endometrial fragments were treated with vehicle, $50 \mu \mathrm{g} / \mathrm{kg}$ per day oral $\mathrm{Cb2}$, or 50 or $200 \mu \mathrm{g} / \mathrm{kg}$ per day quinagolide during a 14-day period. At the end of the treatment period, the implants were excised in order to assess lesion size, cell proliferation, degree of vascularization, and angiogenic gene expression.

Neoangiogenesis was inhibited and the size of active endometriotic lesions, cellular proliferation index, and angiogenic gene expression were significantly reduced by both dopamine agonists when compared with the placebo. Given that $\mathrm{Cb2}$ and quinagolide were equally effective in inhibiting angiogenesis and reducing lesion size, these experiments provide the rationale for pilot studies to explore the use of non-ergot-derived dopamine agonists for the treatment of endometriosis in humans.
\end{abstract}

Reproduction (2011) $\mathbf{1 4 2} 745-755$

\section{Introduction}

Endometriosis is defined as the presence of endometrial tissue, glands, and stroma at sites other than the uterus. As in the case of the eutopic endometrium, the growth of ectopic lesions is hormonally regulated, causing periodic bleeding and inflammation associated with pelvic pain and infertility (Galle 1989).

Numerous peritoneal blood vessels surround active endometriotic lesions, and the implant itself is also endowed with a rich vascularized area; this can be observed under histological examination (Nisolle et al. 1993, Nap et al. 2004) and confirms the essential role that angiogenesis plays in the development and continuation of endometriotic lesions (Shifren et al. 1996, Donnez et al. 1998, Becker et al. 2005). In this regard, it has been observed that human endometrium engrafted in immunocompromised mice promotes the growth of neovessels from the surrounding host vascular network in order to provide the endometriotic lesions with an adequate blood supply. Vascular endothelial growth factor (VEGF) acts through its VEGFR2 to play a pivotal role in the control of angiogenesis (McLaren et al. 1996) and so several antiangiogenic agents that target this pathway have been successfully used to disrupt the vascular supply of heterologous implants in inmmunodeficient animal models of endometriosis (Nap et al. 2004). However, the commercial anti-VEGF/VEGFR2 used in such experiments can induce severe side effects (Eremina et al. 2008), which rules out its use in the treatment of endometriosis in young and otherwise healthy women. Moreover, such compounds can also affect other important physiologic processes, including early pregnancy, by blocking implantation-related ovarian (Wulff et al. 2001, Zimmermann et al. 2001a, 2001b, 2003, Pauli et al. 2005) and uterine angiogenesis (Rockwell et al. 2002, Heryanto et al. 2003). 
We hypothesized that dopamine receptor 2 (DRD2) agonists (DRD2-A) could be an alternative to commercial antiangiogenic agents. In animal models, DRD2-A inhibits pathologic angiogenesis in tumors (Basu et al. 2004) by inactivating VEGFR2 signaling (Basu et al. 2001). However, this type of medication has an acceptable safety profile and does not interfere with the normal establishment and progression of pregnancy (Robert et al. 1996, Ricci et al. 2002). We have previously used a well-established experimental endometriosis model to demonstrate that the DRD2-A cabergoline $(\mathrm{Cb} 2)$ prevents the formation of typical endometriosis lesions by inhibiting angiogenesis (Novella-Maestre et al. 2009). These results encouraged us to perform a pilot study to evaluate the efficacy of $\mathrm{Cb} 2$ in the treatment of peritoneal endometriosis in humans.

An increased incidence of cardiac valve regurgitation has recently been associated with the use of ergotderived DRD2-A such as Cb2 and pergolide in human subjects (Schade et al. 2007, Zanettini et al. 2007). These results have promoted the search for non-ergot-derived DRD2-A drugs with a more benign profile for the treatment of endometriosis.

In order to evaluate the potential benefits of nonergot-derived DRD2-A for the treatment of human endometriosis, we assayed in a heterologous mouse model of endometriosis the efficacy of the DRD2-A quinagolide at 50 and $200 \mu \mathrm{g} / \mathrm{kg}$ doses in preventing angiogenesis and vascularization when compared with placebo or the ergot-derived $\mathrm{Cb} 2$ at $50 \mu \mathrm{g} / \mathrm{kg}$ as a comparator. We also evaluated in these four groups of animals whether or not the VEGF/VEGFR2 pathway was altered during the treatment.

\section{Results}

\section{Evaluation of the endometriosis model}

No toxic response to the treatment or signs of stress as a consequence of the handling and surgical procedures were observed. Similarly, no changes in behavior or significant variation in weight were detected among the different groups.

Of the four endometrial implants introduced per animal, $3.75 \pm 0.25$ were recovered per mouse, but histological analysis revealed the histological hallmarks of endometriosis (glands and stroma) in only $3.5 \pm 0.28$ lesions per animal.

\section{Histological analysis of lesions after $\mathrm{Cb2}$ and quinagolide treatment}

The lesion recovery rate was similar in all the groups studied, and no significant differences were detected among the groups with respect to the number of gland profiles per field in different implants or in the glandular/ stromal area ratio. Both the percentage of active lesions and the lesion size decreased in both DRD2-A-treated groups when compared with controls (Table 1).

\section{Immunohistochemical quantification of proliferation, vascular density, maturity, VEGFR2, and Drd2 expression}

DRD2-A administration reduced proliferation of endometrial implants, which was evident in the significantly lower smaller area of nuclear Ki67 staining in the glands and stroma of the $\mathrm{Cb} 2(P=0.020)$ and the $50 \mu \mathrm{g} / \mathrm{kg}$ $(P=0.029)$ and $200 \mu \mathrm{g} / \mathrm{kg}(P=0.039)$ quinagolide groups with respect to controls (Fig. 1 graph). However, this effect was not associated with a decrease in vascular density in any of the DRD2-A-treated groups (Fig. 2 graph). We did, nevertheless, detect a significant decrease in the number of immature vessels remaining in the DRD2-A-administered groups when compared with controls (Fig. 3 graph). Although no decreases were observed in the area occupied by vessel structures, the activation of DRD2 significantly reduced the area stained by VEGFR2 (Fig. 4 graph). Immunohistochemistry against DRD2 displayed a punctate pattern of staining in single cells and clusters of cells randomly distributed throughout the stroma. Given the fact that most of the 3,3'-diaminobenzidine (DAB; dark brown) staining for DRD2 appeared in hemosiderin deposits (light brown), we suspect that most of the cells expressing this receptor in the endometriotic lesions were macrophages (Fig. 5). The area of tissue stained against DRD2 in the endometriotic lesion tended to be

Table 1 Morphometric analysis of endometriotic-like fragments recovered from nude mice peritoneum in control, cabergoline $50 \mu \mathrm{g} / \mathrm{kg}(\mathrm{Cab})$, quinagolide $50 \mu \mathrm{g} / \mathrm{kg}$ (Quin50), and quinagolide $200 \mu \mathrm{g} / \mathrm{kg}$ (Quin200) high-dose groups.

\begin{tabular}{lccrr}
\hline & Control & Cab & Quin50 & Quin200 \\
\hline Recovery rate (\%) & $93.7 \pm 6.2$ & $87.5 \pm 7.2$ & $93.7 \pm 6.2$ & $93.7 \pm 6.2$ \\
Active lesion (\%) & $93.7 \pm 6.2$ & $47.9 \pm 16.7$ & $54.16 \pm 11.0$ & $60.41 \pm 11.9$ \\
Lesion size (mm $\left.{ }^{2}\right)$ & $9.64 \pm 0.48$ & $7.86 \pm 0.38$ & $7.77 \pm 0.37$ & $6.03 \pm 0.39$ \\
Glands/stroma & $0.24 \pm 0.04$ & $0.22 \pm 0.07$ & $0.19 \pm 0.06$ & $<0.05$ \\
Glands/implant & $5.00 \pm 0.97$ & $5.86 \pm 2.65$ & $4.38 \pm 1.60$ & $0.22 \pm 0.02$ \\
\hline
\end{tabular}

Recovery rate \%, percentage of implanted endometrial fragments that were recovered after the treatments; active lesion \%, percentage of lesions showing the simultaneous presence of glands and stroma. Lesion size was calculated by multiplying the width and the length of the lesions recovered (expressed in $\mathrm{mm}^{2}$ ). Glands/stroma, ratio of the areas occupied by glands and stroma in active lesions; glands/implant, mean number of glands $\times$ microscopic field $(10 \times)$ analyzed in active lesions. Results are expressed as mean \pm s.E.M. 
larger in DRD2-A-treated animals and reached statistical significance in the $200 \mu \mathrm{g} / \mathrm{kg}$ per day quinagolide group (Fig. 5 graph).

\section{Real-time fluorescence-based quantitative PCR}

VEGF, VEFGR2, and Drd2 gene expression was evaluated by RT-PCR in order to detect expression at the mRNA level. Of the three transcripts quantified, VEGFR2 was the only one that exhibited significant statistical differences $(P<0.05)$ in all DRD2-A treated groups (decreased mRNA expression levels) when compared to controls. Surprisingly, VEGF mRNA levels were significantly lower in the quinagolide- but not in the $\mathrm{Cb} 2$-treated groups with respect to controls. We observed a marked tendency for Drd2 mRNA to rise with the higher doses of quinagolide, but these increases were not statistically significant when compared with controls (Fig. 6).

\section{Discussion}

The main goal of this study was to evaluate whether a non-ergot-derived DRD2-A-like quinagolide is as potentially effective as the ergot-derived DRD2-A Cb2 in preventing endometriotic angiogenesis by blocking the VEGF/VEGFR2 system. Interestingly, we found that quinagolide was equally effective as $\mathrm{Cb} 2$ in decreasing lesion size and proliferation and in decreasing VEGFR2 mRNA and VEGF mRNA levels.

We focused our attention on the VEGF/VEGFR2 signaling pathway, as accumulated evidence suggests that DRD2-A binding to the DRD2 expressed on endothelial cells can inactivate VEGFR2 in an autocrine fashion, thereby avoiding its mediated angiogenic actions (Basu et al. 2001). Our results provide the first solid evidence of an alternative mechanism of action of the DRD2-A quinagolide by which it decreases angiogenesis. Indeed, we herein report that quinagolide decreases the expression of VEGF mRNA levels. Based on the punctate expression pattern of DRD2 staining observed in human endometrial implants and our unpublished results using human endometrial biopsies obtained throughout the whole menstrual cycle, we do not believe that this receptor is consistently expressed by the glandular epithelium, stroma, or endometrial vessels. On the contrary, we believe that DRD2 is expressed by cells of an immune origin - perhaps macrophages - and that these cells are probably responsible for inducing, in an autocrine and/or paracrine fashion, a decreased expression of VEGF when activated by DRD2-A. In this context, previous work by our group (Ferrero et al. 2010) and other authors (Cristina et al. 2005) has shown that cell populations expressing DRD2 (granulosa or pituitary cells) inhibit the secretion of VEGF in either an autocrine or a paracrine fashion. In this regard, it is known that most immune cell subtypes express DRD2 (McKenna
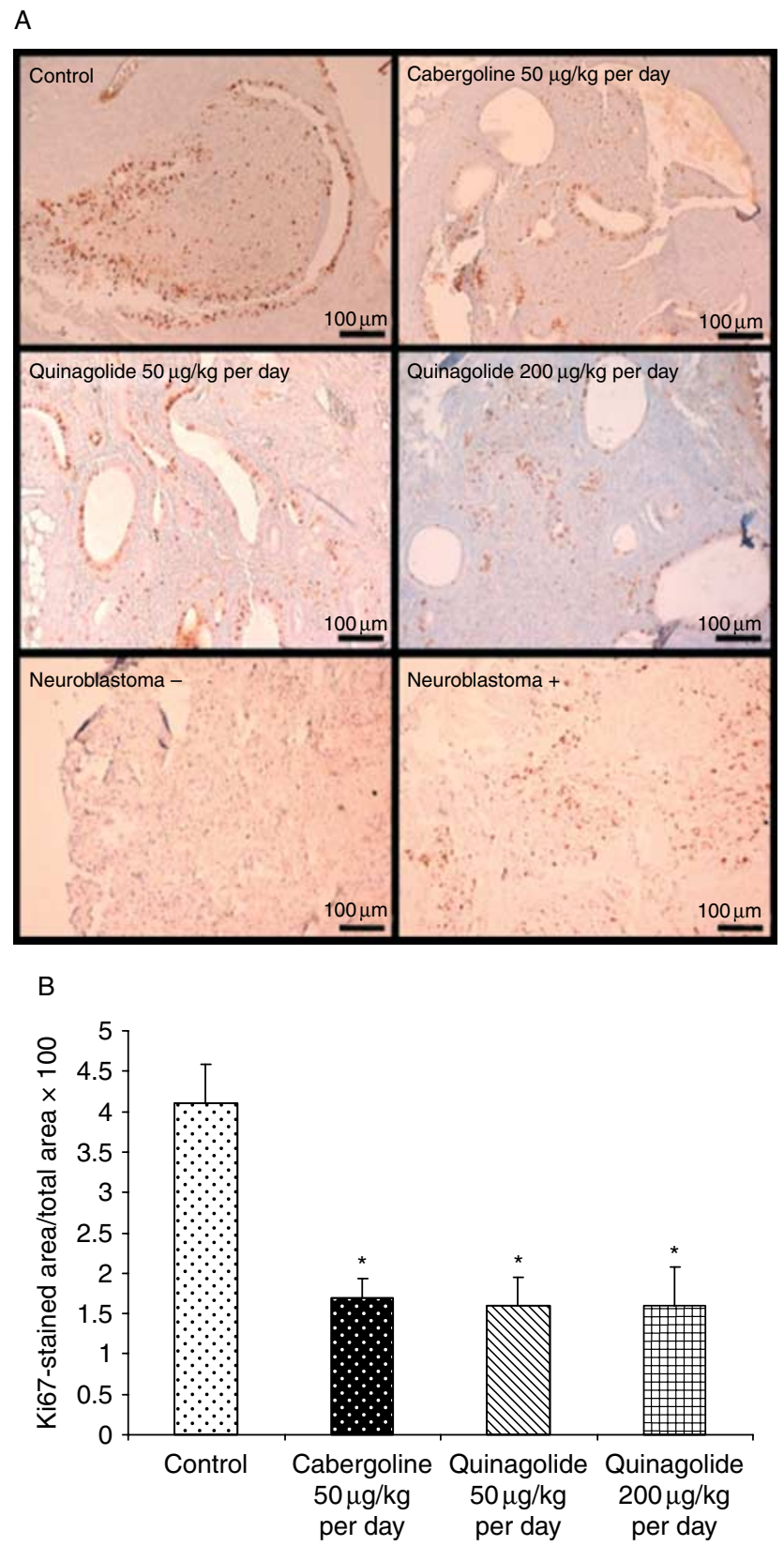

Figure 1 Cell proliferation of implants was investigated using the Ki67 labeling area (A). Representative pictures of immunohistochemical detection of Ki67 (brown color) in endometriotic-like implants from DRD2-A-treated and control mice. Note how cell proliferation is decreased in endometriotic implants of all DRD2-A-treated mice versus controls. Human neuroblastoma was used as a positive control for Ki67. (B) Percentage of stained area represented by Ki67-positive cells per area of interest, $* P<0.05$.

et al. 2002), and among them, macrophages are known to express large amounts of VEGF (Nap et al. 2004). Moreover, the ability of macrophages to reduce angiogenesis when activated by dopamine has recently been described (Chakroborty et al. 2008). Thus, in our experiments, it is likely that quinagolide decreased VEGF transcripts in implanted endometrial tissue by 


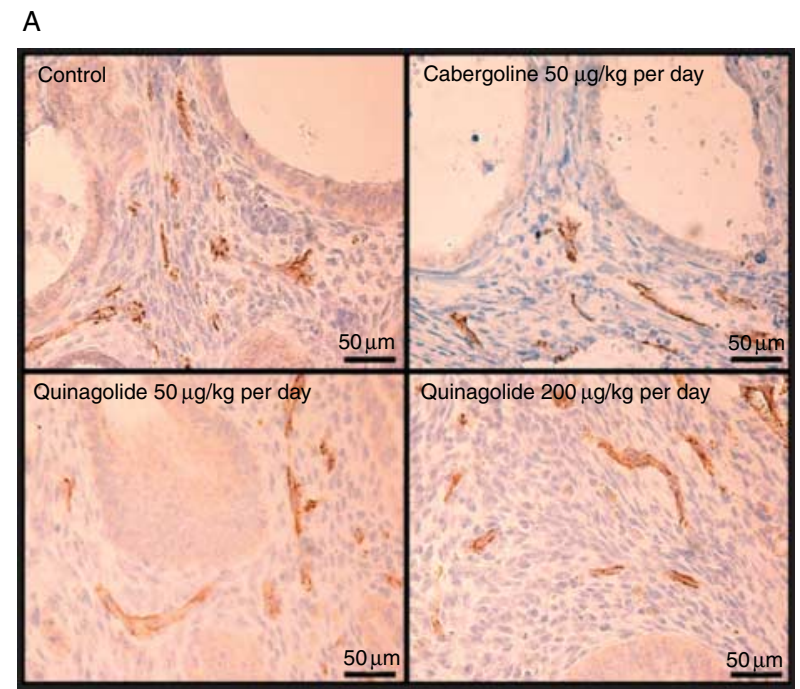

B

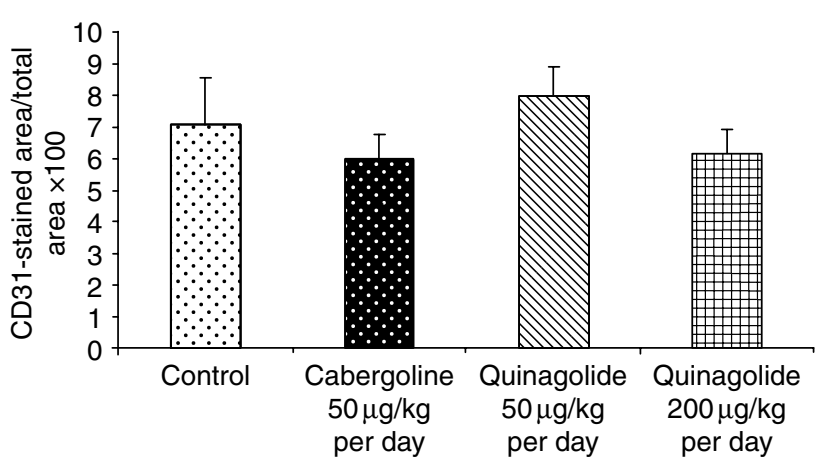

Figure 2 (A) Representative pictures of vascularization in endometrioticlike implants in DRD2-A-treated and control mice. Vessels were immunohistochemically detected using the endothelial marker CD31 (brown color). (B) Microvascular density of endometriotic-like implants in DRD2-A-treated and control mice was determined through

quantification of the area stained by CD31 antigen per area of interest.

inhibiting the expression of this factor in macrophages through the activation of DRD2.

We have also shown that quinagolide decreases the expression of not only VEGF but also VEGFR2 mRNA levels and that this is associated with a reduction in the area of the heterologous implant immunostained against the VEGFR2 protein. In accordance with previously reported data, the pattern of VEGFR2 immunodistribution observed in our endometriosis-like tissue was also exhibited in non-vascular cells, such as those in the glandular epithelium and interstitial stroma (Meduri et al. 2000, Bourlev et al. 2006, Punyadeera et al. 2006, Machado et al. 2008, 2010). Given that DRD2 does not seem to be consistently expressed in VEGFR2positive structures, we hypothesize that the reduction in VEGFR2 expression in cell types expressing this receptor was exerted by DRD2-A in a paracrine fashion.

The growth of newly formed blood vessels is of pivotal importance in the development of endometriosis
(Nap et al. 2004). Angiogenesis involves complex molecular interactions between endometrial tissue, peritoneal tissue, infiltrating leucocytes, and fibroblasts (Hull et al. 2008). In addition, the origin of neovasculature in xenografts remains unclear, which further complicates the analysis of these interactions. Some authors have previously stated that human endometrial tissues transplanted in mice derive their blood supply from the surrounding vascular network, with the original human vessel simultaneously disappearing as the mouse vessels invade the stroma (Grümmer 2001, Bruner-Tran et al. 2002, Hull et al. 2003, Eggermont et al. 2005). However, recent studies have revealed the presence of mouse, human, and functional chimeric human-mouse vessels throughout such grafts (Masuda et al. 2007,

A
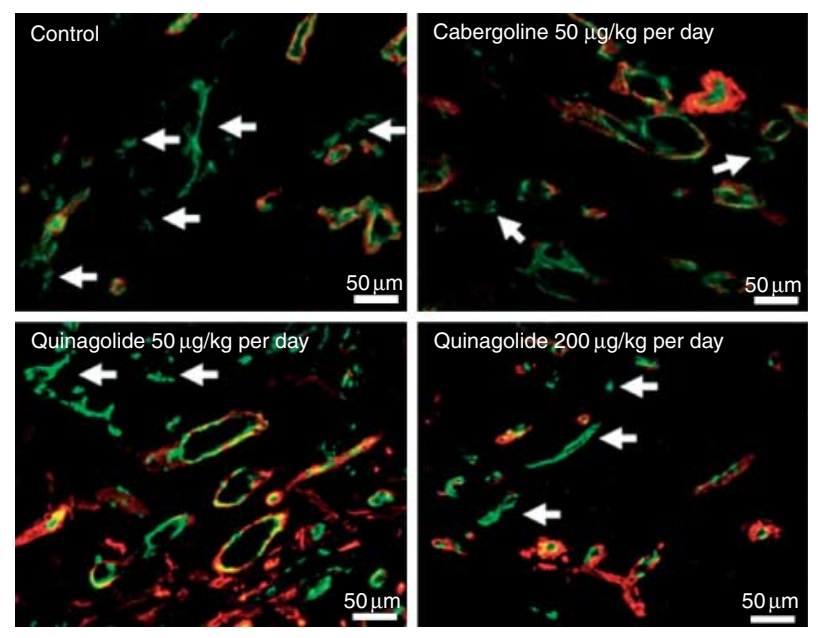

B

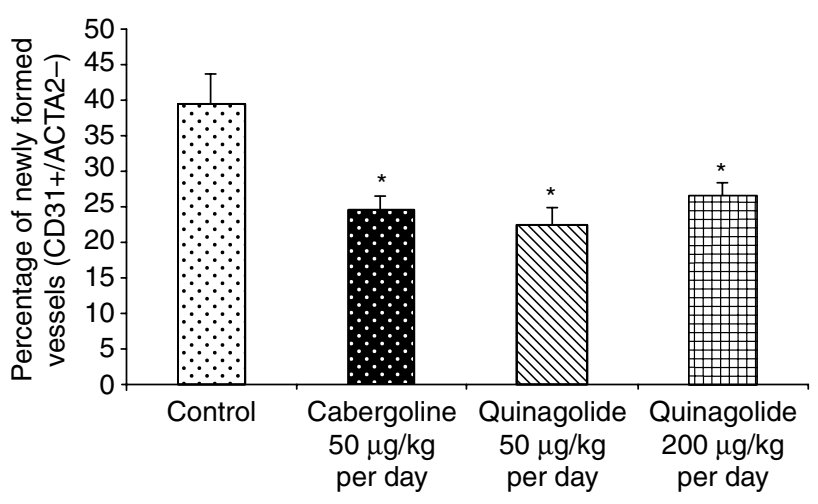

Figure 3 Effects of dopamine agonists on neoangiogenesis.

(A) Representative pictures of double immunofluorescence for CD31 (green) and alpha smooth muscle actin (ACTA2, also known as $\alpha$-SMA; red) in endometriotic-like implant sections from controls and DRD2-Atreated mice. White arrows point to immature vessels showing staining for CD31 (green) but lacking staining for ACTA2 (red). Mature vessels show double staining for CD31 and ACTA2. (B) Percentage of newly formed vessels in each group, represented as immature vessels per field. $* P<0.05$. 
A

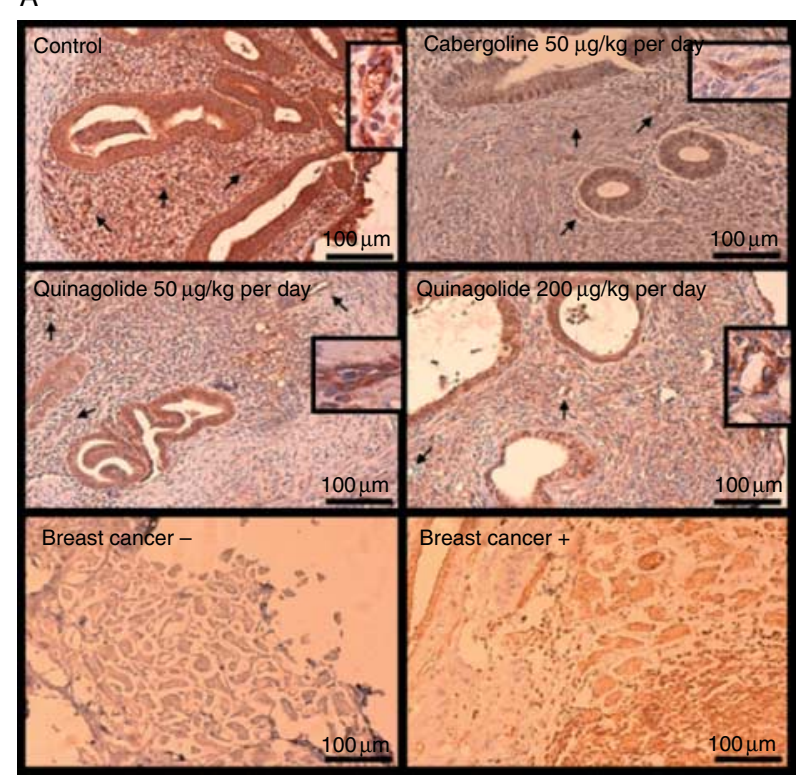

B

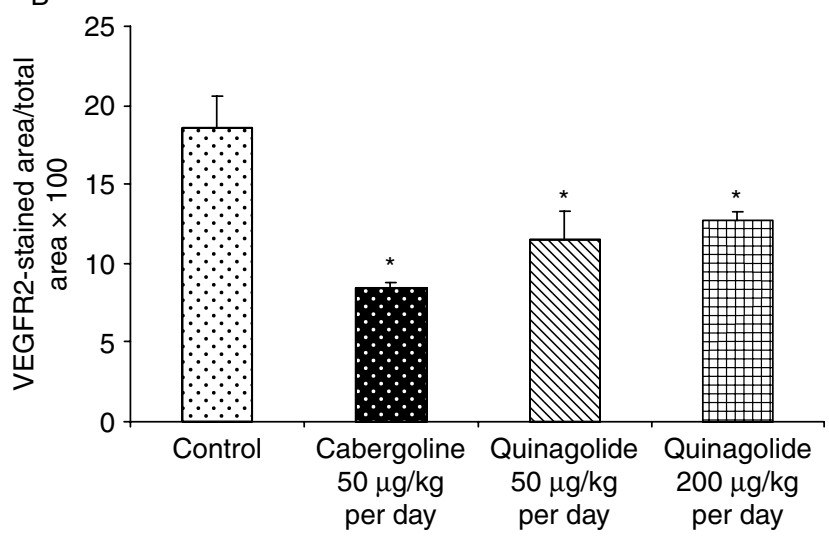

Figure 4 Effects of dopamine agonists on VEGFR2 expression.

(A) Representative pictures of immunohistochemical detection of VEGFR2 (brown) in endometriotic-like implants from DRD2-A-treated and control mice. VEGFR2 staining was observed in stroma, glands, and blood vessels (black arrows). Detailed VEGFR2 staining in vessel structures in representative images from each group is shown in a small box. Note the decreased expression of VEGFR2 in DRD2-A-treated versus control groups. (B) Estimation of VEGFR2 expression through quantification of VEGFR2-stained area per area of interest in DRD2treated and control groups. ${ }^{*} P<0.05$.

Álvarez-González et al. 2009, Guo et al. 2011). Similarly, host-derived cell types, such as macrophages and myofibroblasts, have been shown to coexist with macrophages from human endometrium embedded in the stroma of immunocompromised mouse xenografts (Hull et al. 2008, Guo et al. 2011). Such studies reveal an interaction between host and graft at different levels, by which a combined structure capable of responding to different stimuli is created. In the context of this interaction, the objective of our study was to evaluate the angiogenic response as a holistic process in which both human and mouse tissues are implicated. Thus, antibodies and primers were selected to determine both human and mouse contributions, respectively, thereby obtaining a wider perspective of this process.

As was the case in previous experiments performed in the same heterologous animal model of endometriosis and in which we assayed different doses of $\mathrm{Cb} 2$ (Novella-Maestre et al. 2009), we observed no reduction in the area occupied by vessels but did find a decrease in the number of newly formed vessels in response to DRD2-A. We speculate that this behavior is a result of mature 'major' vessels being less sensitive to the actions
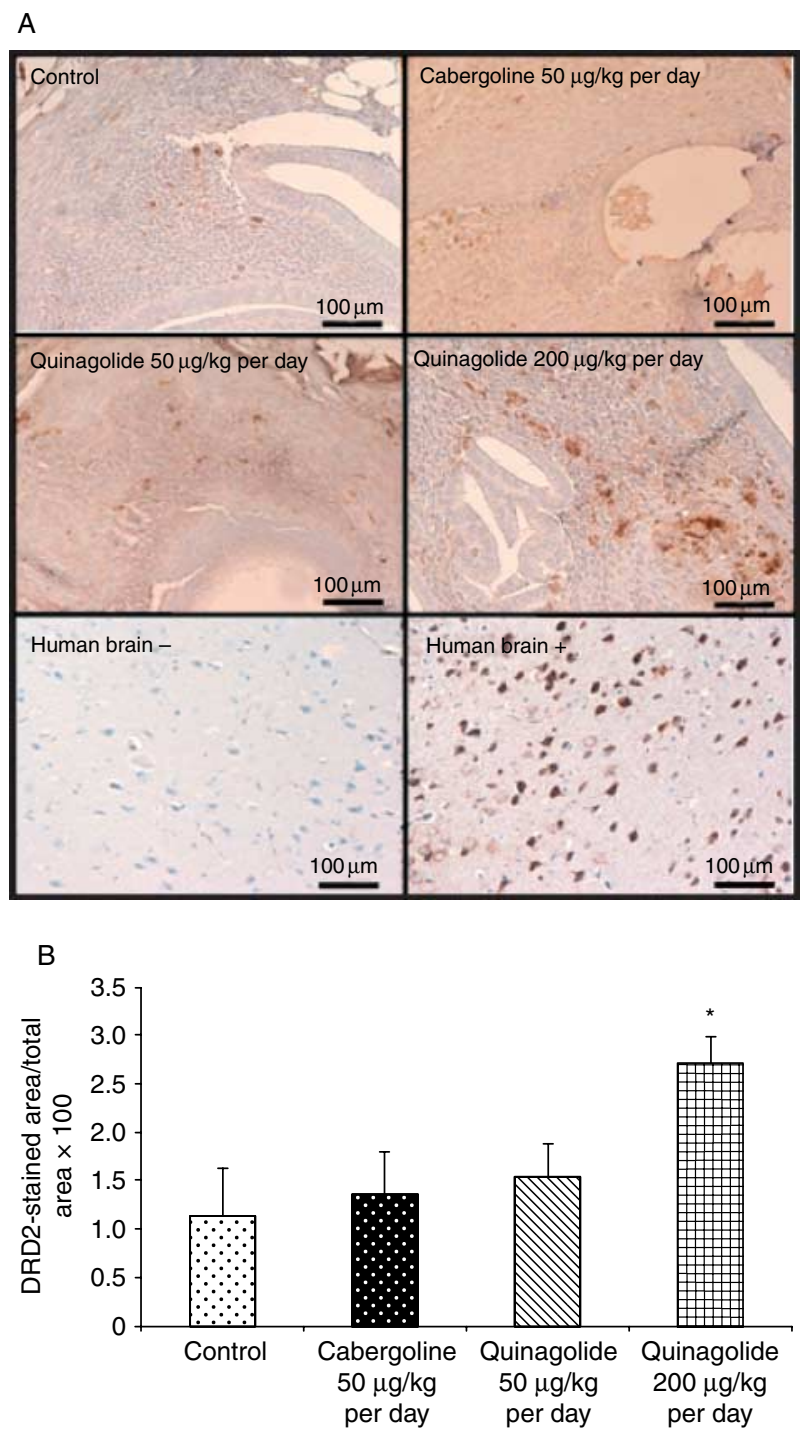

Figure 5 Effects of dopamine agonists on DRD2 expression. (A) Representative pictures of immunohistochemical detection of DRD2 (brown) in endometriotic-like implants from DRD2-A-treated and control mice. DRD2-A showed a punctate immunohistochemical pattern probably associated with scattered macrophages going through the stroma. Note the increased expression of DRD2 in DRD2-A-treated versus controls groups. (B) Estimation of DRD2 expression through quantification of DRD2-stained area per area of interest in DRD2treated and control groups. ${ }^{*} P<0.05$. 

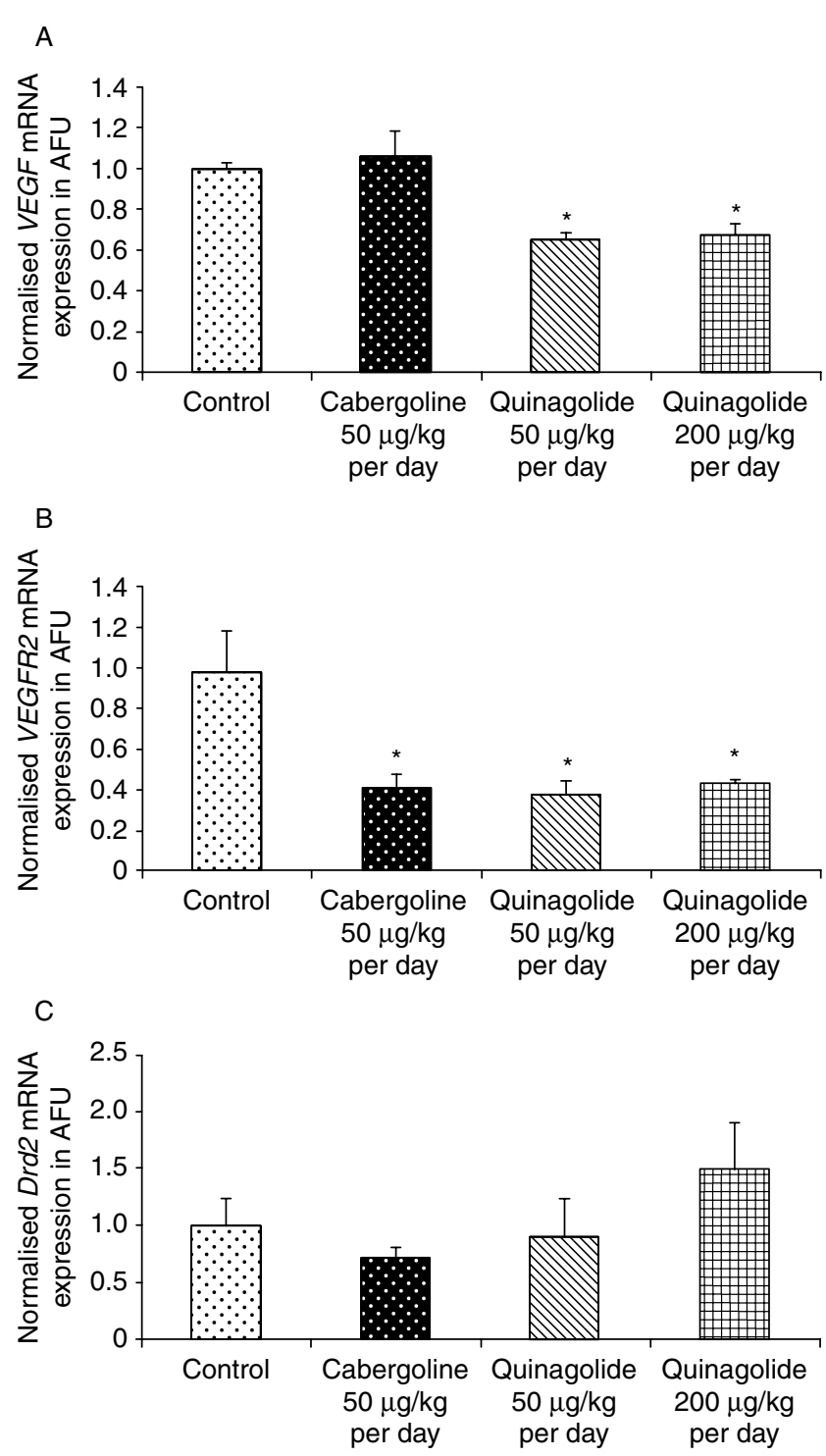

Figure 6 Comparison of (A) VEGF, (B) VEGFR2, and (C) Drd2 mRNA levels in quinagolide- and cabergoline-treated versus control animal groups, determined by QF-PCR. AFU, arbitrary fluorescence units. $* P<0.05$.

of VEGF due to their pericyte (ACTA2 +) coverage, while newly formed 'small' vessels, which are free of pericytes, are more sensitive to VEGF (Benjamin et al. 1998). As DRD2-A reduced VEGF mRNA levels, it is feasible that quinagolide and $\mathrm{Cb} 2$ inhibited the revascularization process, which took place in the engrafted endometrium. As the specific origin of newly proliferating cells is unknown, we used antibodies to detect both human and murine blood vessel markers in order to analyze the contribution of both species to the hypothetic antiangiogenic effect of DRD2. We observed a slight shrinkage in the size of peritoneal lesions treated with DRD2-A. Thus, we cannot rule out that the reduction in the vascularized area induced by DRD2-A, by taking place in vanished/regressed tissue, even in mature vessels, was masked. In fact, a significant decrease in the proliferation index induced by DRD2-A, as shown by decreased Ki67 staining, suggested that effects of $\mathrm{Cb} 2$ and quinagolide, which were inhibitory for angiogenesis, were ongoing in the xenograft. We speculate that by increasing the period of $\mathrm{Cb} 2$ and quinagolide treatment in the nude model, it may be possible to detect decreases in vascularization. However, due to the characteristics of the animal model used and the constraints of time in this study, the prolonged survival of implanted tissue was not possible.

Despite strong evidence to suggest that DRD2 activation modulates angiogenesis by directly interfering with VEGFR2 signaling (Basu et al. 2001), it is plausible that dopamine agonists also inhibit angiogenesis in ectopic implants through alternative mechanisms. In this regard, and given that prolactin (PRL) secretion has been shown to promote angiogenesis by signaling through specific blood vessel receptors (Reese et al. 2000), it is possible that the reduction of circulating PRL levels as a result of DRD2 also contributes to the inhibition of the angiogenic response induced by these drugs in endometrial implants. Although there are several reports of an association between endometriosis and hyperprolactinemia (Gregoriou et al. 1999, Cunha-Filho et al. 2002), the existence of a fundamental proangiogenic role for PRL in human endometrial ectopic tissue is yet to be confirmed.

We did not evaluate whether DRD2-A affected angiogenesis in the reproductive organs, but such an action is unlikely in light of previous results published by our group after using tenfold higher DRD2-A doses than those used in this study, which showed that luteal angiogenesis and function were not compromised (Gómez et al. 2006). Further evidence of the inexistence of the detrimental effects of DRD2-A for eutopic endometrial angiogenesis is provided by the fact that women receiving high doses of $\mathrm{Cb} 2$ become pregnant uneventfully (Verhelst et al. 1999). Given its different mechanism of action, DRD2-As are unlikely to interfere with the menstrual cycle and thus possesses an important advantage over GNRH agonists and aromatase inhibitors, which induce osteoporosis and hot flushes as a result of hypoestrogenic milieu (Gregoriou et al. 1997, Walch et al. 2009).

In conclusion, the successful inhibition of ectopic endometrial growth by both types of DRD2-A under study in a immunocompromised mouse model provides the rationale for a pilot study in humans. Our results suggest that quinagolide is as effective as Cb2 in inhibiting the growth of endometriotic lesions in endometriosis patients. Moreover, as quinagolide is not an ergot derivate, it offers an additional advantage over $\mathrm{Cb} 2$, as it does not produce the side effects that ergot derivatives exert on the serotonin (5-hydroxytryptamine (5-HT)) receptor subtype 5-HT2b. Unlike Cb2, quinagolide is unlikely to affect cardiac valve tissue and, 
therefore, is not expected to induce proliferation of fibroblasts (Roth 2007) or regurgitation. In addition, quinagolide has a much shorter half-life $(\sim 17 \mathrm{~h})$ than Cb2 ( 63-69 h; Colao et al. 2002), thus minimizing exposure during organogenesis for women who wish to become pregnant while under treatment with DRD2-A. Inducing a hyperprolactinemic state in the event of DRD2-A proving ineffective in the treatment of women with endometriosis may be ethically questionable. To avoid such controversy, we propose assaying these drugs in women suffering simultaneously from endometriosis and hyperprolactinemia, as DRD2-A therapy is indicated for both these conditions.

\section{Materials and Methods}

This study was approved by our Institutional Review Board and the informed consent of subjects was obtained prior to endometrial biopsy collection. Similarly, all the procedures using animals were performed according to the European Directive 86/609/CEE and NIH Guidelines for the Care and Use of Laboratory Animals.

\section{Experimental model of endometriosis}

The model of endometriosis was prepared as described previously (Nap et al. 2004) with minor modifications. A total of 34 ovariectomized 5-week-old female mice (Hsd: athymic Nude-nu; Harlan Ibérica S.L, Barcelona, Spain) were individually housed in autoclaved cages (with bedding) in laminar flow filtered hoods. The animal room was maintained at $26^{\circ} \mathrm{C}$ with a $12 \mathrm{~h}$ light: $12 \mathrm{~h}$ darkness cycle, and mice were fed with autoclaved laboratory rodent chow and acidified water ad libitum. All handling was performed in laminar flow filtered hoods. A mixture of $75 \mathrm{mg} / \mathrm{g}$ ketamine (Ketolar, ParkeDavis, Barcelona, Spain) and $1 \mathrm{mg} / \mathrm{g}$ medetomidine (Domtor, Pfizer, Madrid, Spain) was injected i.p. to anesthetize mice before invasive procedures. Atipamezole $(1 \mathrm{mg} / \mathrm{g}$; Antisedan, SmithKline Beecham, Madrid, Spain) was administered i.p. after invasive procedures to reverse the effects of anesthesia. Sixtyday-release sterile capsules containing $18 \mathrm{mg} 17 \beta$-estradiol (Innovative Research of America, Sarasota, FL, USA) were placed s.c. in the neck of each animal. Four days later, fresh human endometrium fragments were acquired via biopsy during ovum retrieval from four oocyte donors with normal menstrual cycles and no history of endometriosis (18-34 years old). These fragments, after being minced into pieces of $\sim 3 \times 3 \mathrm{~mm}$, were attached to the wall of the peritoneal cavity of each mouse using $n$-butyl-ester cyanoacrylate adhesive (Vetbond; 3M Animal Care Products, St Paul, MN, USA). Human endometrial samples from individual donors were used in at least two animals from each experimental group; in this way, four implants were introduced per animal. Three weeks after establishment of lesions, the animals were divided into four groups: three experimental and one control. In the experimental groups, $50 \mu \mathrm{g} / \mathrm{kg}$ per day of $\mathrm{Cb} 2$ (Dostinex, Pfizer Labs) or 50 or $200 \mu \mathrm{g} / \mathrm{kg}$ per day of quinagolide (Norprolac, Ferring Pharmaceuticals) were administered by oral gavage for
14 days. The dose of $\mathrm{Cb} 2$ that we used has previously been shown to be effective in inhibiting angiogenesis in the same endometriosis mouse model (Novella-Maestre et al. 2009) and was used as a reference for selecting the quinagolide doses assayed. The $50 \mu \mathrm{g} / \mathrm{kg}$ dose of quinagolide was chosen in order to evaluate the effects of this drug when administered at the same daily doses as $\mathrm{Cb} 2$. Given that $\mathrm{Cb} 2$ has a higher half-life than quinagolide (Colao et al. 2002), the $200 \mu \mathrm{g} / \mathrm{kg}$ dose of quinagolide was selected in an attempt to compare the effects exerted by similar accumulative doses of both compounds by the end of the treatment. Animals in the control group were administered with vehicle solution. The animals were killed by cervical dislocation $24 \mathrm{~h}$ after the last administration of drug or vehicle, and the abdominal skin and peritoneum were opened to examine the visceral organs under a binocular microscope and thus evaluate the presence of endometriotic implants and vascularization. The size of all the lesions in the peritoneum of mice with possible endometriosis was estimated, as described below. One half of the animals $(n=17)$ were used for microscopic histological, immunohistochemical, and subsequent morphometric quantitative studies, and the remaining half $(n=17)$ underwent the same procedures to obtain tissue, which was frozen at $-80^{\circ} \mathrm{C}$ for subsequent molecular analysis of angiogenesis-related genes.

\section{Macroscopic analysis}

Before harvesting the implants from the peritoneum, they were counted and a vernier calliper was used to measure their length and width. Lesion size was calculated by multiplying the length and width and was expressed in $\mathrm{mm}^{2}$.

\section{Histologic studies}

Tissues were fixed in $4 \%$ neutral buffered formalin overnight at $48{ }^{\circ} \mathrm{C}$ before being embedded in paraffin wax for histological and immunohistochemical analyses. Four to five non-contiguous $4 \mu \mathrm{m}$ sections from each specimen were stained with hematoxylin-eosin (Sigma Co.) and examined microscopically for histological hallmarks (glands and stroma) of endometriosis. Following the histological study, only the lesions that contained both glandular and stromal elements were considered to be active. Those presenting an atrophic epithelium surrounded by fibrotic tissue rather than stroma were considered non-active lesions and were discarded from the study.

\section{Immunohistochemistry}

Standard immunohistochemistry was achieved using specific primary antibodies for each determination. Proliferating cells were detected by using a mouse MAB against Ki67 (MIB-1, 1:50; DakoCytomation, Glostrup, Denmark). The blood vessel supply of the endometrial implants was determined using a rabbit polyclonal antibody against CD31 (ab28364, 1:200, Abcam, Cambridge, MA, USA), which labels endothelial cells from both micro- and macrovasculature. A mouse MAB for VEGFR2 (sc-6251, 1:50; Santa Cruz Biotechnology, Inc., Santa Cruz, CA, USA) and a mouse MAB for DRD2 (sc-5303, 1:50; Santa Cruz Biotechnology, Inc.) were applied to detect the 
presence of these receptors in the human endometrium engraftment in the animals' peritonea.

Four micron serial sections were deparaffinized and rehydrated through graded ethanol and rinsed in distilled water. Antigen retrieval was carried out by the autoclave method $\left(120^{\circ} \mathrm{C}\right.$ ) for $3 \mathrm{~min}$ in $0.5 \mathrm{M}$ Tris buffer at $\mathrm{pH} 10$ (for CD31; Wang et al. 2008) or $0.01 \mathrm{M}$ citrate buffer at $\mathrm{pH} 6.0$ (for Ki67, VEGFR2 and DRD2) followed by cooling for $20 \mathrm{~min}$ at room temperature. Sections were gently rinsed in deionized water and then transferred to a $0.5 \mathrm{M}$ Tris-based solution in $0.15 \mathrm{M} \mathrm{NaCl}$ with $0.1 \% \mathrm{v} / \mathrm{v}$ Triton-X-100 at $\mathrm{pH} 7.6$ (TBST). Endogenous peroxidase was blocked for $10 \mathrm{~min}$ with $3 \%$ hydrogen peroxide. To further reduce non-specific background staining, slides were incubated at room temperature with an avidin/biotin blocking kit (Vector Labs, Burlingame, CA, USA). The sections were incubated overnight with the primary antibodies in a humidified chamber at $4{ }^{\circ} \mathrm{C}$. After washing with TBST, sections were incubated with biotinylated goat antirabbit IgG (B8895, 1:800; Sigma-Aldrich Ltd) for CD31, or secondary link biotinylated antibody (K0679, LSAB + kit; Dakocytomation) for Ki67, VEGFR2, and DRD2 for $30 \mathrm{~min}$ at room temperature and then with streptavidin-HRP for $30 \mathrm{~min}$, using 3,3'-diaminobenzidine as the chromogen (K0679, $\mathrm{LSAB}+$ kit; Dakocytomation). Sections were then counterstained with Mayer's hematoxylin and then dehydrated and mounted in mounting medium (Eukitt 15 320).

The following positive controls were used in the immunohistochemistry assay: neuroblastoma for Ki67, human breast cancer for VEGFR2, human endometrium positive for CD31, and human brain for DRD2. With the exception of ACTA2, for which a non-specific IgG antibody was used, negative controls were performed by omission and pre-absorption of the primary antibodies with a specific immunogen peptide acquired from the same company that supplied us with the primary antibodies.

\section{Immunofluorescence}

In order to determine whether vessels supporting the lesions were mature or immature, we performed a dual immunofluorescence procedure by detecting pericytes with an ACTA2 antibody (C6198. 1:500; Sigma-Aldrich Ltd) directly labeled with Cy3 in sections previously stained with CD31. We did this due to the fact that pericytes surround mature vessels but are absent in new proliferating immature vessels.

Detection of immunofluorescence was performed in the same way as immunohistochemistry, but using a Tyramide Signal Amplification Fluorescein system (NEL701A001KT, TSA; Perkin Elmer Life Sciences, Boston, MA, USA) instead of $\mathrm{DAB}$, following the manufacturer's protocol. Finally, the slides were rinsed in PBS and counterstained with mounting medium ProLong Gold antifade reagent with DAPI (Invitrogen, P36931).

\section{Morphometric analysis}

High-quality images $(2048 \times 1536$ pixels $)$ from histological and immunostained sections were recorded randomly with an Olympus BH2-UMA microscope connected to a JVC/TK-1270 video camera and a computer-digitized plate (Olympus, Tokyo, Japan). The images were processed with
Image ProPlus 6.0 Software (Media Cybernetics, Silver Springs, MD, USA) in order to manually delineate regions of interest that were free of gaps and detached tissue. Subsequently, the area of interest and the stained area (to be analyzed) were manually or automatically segmented from the region of interest using the same software. Parameters of interest were estimated as follows: at least ten images of each lesion $(10 \times$ field) were used to manually delimitate the glandular and stromal area and were expressed as a ratio of said area. The gland profile was achieved by counting the number of glands appearing per implant in each image $(10 \times$ field) of a lesion. For every immunohistochemical parameter evaluated (except for the quantification of vessel maturity), three non-contiguous slides were photographed in four random high-power $(40 \times)$ fields and processed with Image-pro plus 6.3 to automatically outline, highlight, and quantify the stained areas. The percentage of vascularization, as well as the overall distribution of DRD2 and VEGFR2, was estimated by quantifying the area stained by CD31 (endothelial cell marker), DRD2, and VEGFR2 antibodies per area of interest, according to previously described methods (Caulet et al. 1992, Moody et al. 2004). We initially aimed to evaluate the proliferation index by quantifying the number Ki67 (proliferation marker)-positive cells per total cell number in each area of interest. However, because of the diffuse (brown) staining, the software failed to automatically segment the number of brown-stained nuclei in the quantification of this parameter. Thus, we decided to evaluate proliferation by also quantifying the area stained by Ki67 per area of interest. Despite not being as accurate as counting individual proliferating cells, quantifying the Ki67-stained area is also a trustable and accepted methodology for the estimation of proliferation indexes, which has been previously used by other investigators (Caulet et al. 1992, Belur et al. 2011). Vessel maturity was determined by the operator, who manually counted the number of mature $(\mathrm{CD} 31+/ \mathrm{ACTA} 2+)$ or immature $(\mathrm{CD} 31+/ \mathrm{ACTA} 2-)$ vessels in each area of interest.

\section{Quantitative real-time PCR}

\section{RNA extraction}

Lesions destined for PCR amplification were excised and maintained at $-196{ }^{\circ} \mathrm{C}$ with $100-500 \mu$ l Trizol reagent until ready for processing. The Chomczynski and Sacchi method was used for RNA extraction. The amount of mRNA was quantified in a nanodrop and its integrity assessed with an Agilent 2100 Bioanalyzer (Agilent, Waldbronn, Germany).

\section{Primer design}

Given that the objective of our study was to evaluate angiogenesis in a process in which both human and mouse tissues were implicated, the primers for VEGF, VEGFR2, and Drd2 and the housekeeping gene $\beta$-actin were designed to simultaneously recognize both human and mouse transcripts. Gene sequences were obtained from GenBank (www.ncbi.nlm.nih.gov/entrez/ query. $\mathrm{f}$ cgi $\mathrm{db}=$ nucleotide) and a blast search was performed to ensure that designed primers exhibited $100 \%$ homology 
with both mouse and human sequences while avoiding cross alignment with other unspecific sequences. Primers were purchased from Invitrogen and Bonsai Technologies (Madrid, Spain). The oligonucleotide sequences used for amplification are summarized in Supplementary Table 1, see section on supplementary data given at the end of this article.

\section{$R T-Q F-P C R$}

Total RNA ( $1 \mu \mathrm{g}$ ) was reverse transcribed using an Advantage RT-for-PCR kit (Clontech) and the product was diluted in water to a final volume of $100 \mu \mathrm{l}$.

The amplification of cDNA by quantitative fluorescence (QF)-PCR was performed with the LightCycler-Fast-Start DNA Master SYBR Green I kit (Roche Diagnostics) in a Roche Light Cycler (Roche), following the manufacturer's instructions. cDNA (100 ng) was used for each sample analyzed. A calibration curve was drawn by including six serial dilutions $(1 / 10)$ of a high cDNA concentration sample of each gene in order to interpolate the results in each experiment. The final products were analyzed with the software provided by the manufacturer (Roche Molecular Biochemicals Light Cycler Software v3.5). Human-mouse $\beta$-actin was used as a housekeeping gene to normalize the expression levels of $V E G F$, VEGFR2, and Drd2. Results were expressed as arbitrary fluorescence units after normalizing the ratio (gene of interest/house keeping gene) to the values obtained in the control groups (assigned value $=1$ ).

\section{Statistical analysis}

Statistical analysis was carried out with SPSS (release 15.0 SPSS, Inc., Chicago, IL, USA). Results are expressed as mean + S.E.M., except when specified. The Kolmogorov-Smirnoff test was used to determine whether data followed a normal distribution. Non-parametric Mann-Whitney and Dunn's multiple comparisons test were used to compare individual means. The level of statistical significance was set at $P<0.05$.

\section{Supplementary data}

This is linked to the online version of the paper at http://dx.doi. org/10.1530/REP-11-0223.

\section{Declaration of interest}

The authors declare that there is no conflict of interest that could be perceived as prejudicing the impartiality of the research reported.

\section{Funding}

Supported by grant SAF2007-65334 from the Spanish government, a Lilly Foundation Grant for Research in Clinical Medicine and a grant awarded by Ferring Pharmaceuticals to F Delgado-Rosas.

\section{References}

Álvarez González ML, Galant C, Frankenne F, Nisolle M, Labied S, Foidart JM, Marbaix E \& Béliard A 2009 Development of an animal experimental model to study the effects of levonorgestrel on the human endometrium. Human Reproduction 24 697-704. (doi:10.1093/humrep/ den437)

Basu S, Nagy JA, Pal S, Vasile E, Eckelhoefer IA \& Bliss VS 2001 The neurotransmitter dopamine inhibits angiogenesis induced by vascular permeability factor/vascular endothelial growth factor. Nature Medicine 7 569-574. (doi:10.1038/87895)

Basu S, Sarkar C, Chakroborty D, Nagy J, Mitra RB, Dasgupta PS \& Mukhopadhyay D 2004 Ablation of peripheral dopaminergic nerves stimulates malignant tumor growth by inducing vascular permeability factor/vascular endothelial growth factor-mediated angiogenesis. Cancer Research 64 5551-5555. (doi:10.1158/0008-5472.CAN-04-1600)

Becker CM, Sampson DA, Rupnick MA, Rohan RM, Efstathiou JA, Short SM, Taylor GA, Folkman J \& D'Amato RJ 2005 Endostatin inhibits the growth of endometriotic lesions but does not affect fertility. Fertility and Sterility 84 1144-1155. (doi:10.1016/j.fertnstert.2005.04.040)

Belur LR, Podetz-Pedersen KM, Sorenson BS, Hsu AH, Parker JB, Carlson CS, Saltzman DA, Ramakrishnan S \& Mclvor RS 2011 Inhibition of angiogenesis and suppression of colorectal cancer metastatic to the liver using the Sleeping Beauty Transposon System. Molecular Cancer 10 14. (doi:10.1186/1476-4598-10-14)

Benjamin LE, Hemo I \& Keshet E 1998 A plasticity window for blood vessel remodelling is defined by pericyte coverage of the preformed endothelial network and is regulated by PDGF-B and VEGF. Development 125 1591-1598.

Bourlev V, Volkov N, Pavlovitch S, Lets N, Larsson A \& Olovsson M 2006 The relationship between microvessel density, proliferative activity and expression of vascular endothelial growth factor-A and its receptors in eutopic endometrium and endometriotic lesions. Reproduction 132 501-509. (doi:10.1530/rep.1.01110)

Bruner-Tran KL, Webster-Clair D \& Osteen KG 2002 Experimental endometriosis: the nude mouse as a xenographic host. Annals of the New York Academy of Sciences 955 328-339. (doi:10.1111/j.17496632.2002.tb02793.x)

Caulet S, Lesty C, Raphael M, Schoevaert D, Brousset P, Binet JL, Diebold J \& Delsol G 1992 Comparative quantitative study of Ki-67 antibody staining in $78 \mathrm{~B}$ and $\mathrm{T}$ cell malignant lymphoma (ML) using two image analyser systems. Pathology, Research and Practice 188 490-496.

Chakroborty D, Chowdhury UR, Sarkar C, Baral R, Dasgupta PS \& Basu S 2008 Dopamine regulates endothelial progenitor cell mobilization from mouse bone marrow in tumor vascularization. Journal of Clinical Investigation 118 1380-1389. (doi:10.1172/JCI33125)

Colao A, di Sarno A, Pivonello R, di Somma C \& Lombardi G 2002 Dopamine receptor agonists for treating prolactinomas. Expert Opinion on Investigational Drugs 11 787-800. (doi:10.1517/13543784.11.6. 787)

Cristina C, Díaz-Torga G, Baldi A, Góngora A, Rubinstein M, Low MJ \& Becú Villalobos D 2005 Increased pituitary vascular endothelial growth factor-A in dopaminergic D2 receptor knockout female mice. Endocrinology 146 2952-2962. (doi:10.1210/en.2004-1445)

Cunha-Filho JS, Gross JL, Lemos NA, Dias EC, Vettori D, Souza CA \& Passos EP 2002 Prolactin and growth hormone secretion alter thyrotrophin-releasing hormona infusion and dopaminergic (DA2) blockade in infertile patients with minimal/mild endometriosis. Human Reproduction 17 960-965. (doi:10.1093/humrep/17.4.960)

Donnez J, Smoes P, Gillerot S, Casanas-Roux F \& Nisolle M 1998 Vascular endothelial growth factor in endometriosis. Human Reproduction 13 1686-1690. (doi:10.1093/humrep/13.6.1686)

Eggermont J, Donnez J, Casanas-Roux F, Scholtes H \& Van Langendonckt A 2005 Time course of pelvic endometriotic lesion revascularization in a nude mouse model. Fertility and Sterility 84 492-499. (doi:10.1016/j. fertnstert.2005.03.034)

Eremina V, Jefferson JA, Kowalewska J, Hochster H, Haas M, Weisstuch J, Richardson C, Kopp JB, Kabir MG, Backx PH et al. 2008 VEGF inhibition and renal thrombotic microangiopathy. New England Journal of Medicine 358 1129-1136. (doi:10.1056/NEJMoa0707330)

Ferrero H, Gaytán F, Delgado-Rosas F, Gaytán M, Gómez R, Simón C \& Pellicer A 2010 Dopamine receptor 2 (drd2) activation inhibits VEGF 
secretion in granulosa luteinized cells: implications for OHSS treatment in low drd2 milieus. Human Reproduction 25 (Supplement 1) i56-i58. (doi:10.1093/humrep/de.25.s1.40)

Galle PC 1989 Clinical presentation and diagnosis of endometriosis. Obstetrics and Gynecology Clinics of North America 16 29-42.

Gómez R, González-Izquierdo M, Zimmermann RC, Novella-Maestre E, Alonso-Muriel I, Sanchez-Criado J, Remohi J, Simon C \& Pellicer A 2006 Low-dose dopamine agonist administration blocks vascular endothelial growth factor (VEGF)-mediated vascular hyperpermeability without altering VEGF receptor 2-dependent luteal angiogenesis in a rat ovarian hyperstimulation model. Endocrinology 147 5400-5411. (doi:10.1210/ en.2006-0657)

Gregoriou O, Konidaris S, Vitoratos N, Papadias C, Papoulias I \& Chryssicopoulos A 1997 Gonadotropin releasing hormone analogue plus hormone replacement therapy for the treatment of endometriosis: a randomized controlled trial. International Journal of Fertility and Women's Medicine 42 406-411.

Gregoriou G, Bakas P, Vitoratos N, Papadias K, Goumas K, Chryssicopoulos A \& Creatsas G 1999 Evaluation of serum prolactin levels in patients with endometriosis and infertility. Gynecologic and Obstetric Investigation 48 48-51. (doi:10.1159/000010133)

Grümmer R 2006 Animals models in endometriosis research. Human Reproduction Update 5 641-649. (doi:10.1093/humupd/dml026)

Guo Y, He B, Xu X \& Wang J 2011 Comprehensive analysis of leukocytes, vascularization and matrix metalloproteinases in human menstrual xenograft model. PLOS ONE 6 e16840. (doi:10.1371/journal.pone. 0016840)

Heryanto B, Lipson KE \& Rogers PA 2003 Effect of angiogenesis inhibitors on oestrogen-mediated endometrial endothelial cell proliferation in the ovariectomized mouse. Reproduction 125 337-346. (doi:10.1530/rep.0. 1250337)

Hull ML, Charnock-Jones DS, Chan CLK, Bruner-Tran KL, Osteen KG, Tom BD, Fan TP \& Smith SK 2003 Antiangiogenic agents are effective inhibitors of endometriosis. Journal of Clinical Endocrinology and Metabolism 88 2889-2899. (doi:10.1210/jc.2002-021912)

Hull ML, Escareno CR, Godsland JM, Doig JR, Johnson CM, Phillips SC, Smith SK, Tavaré S, Print CG \& Charnock-Jones DS 2008 Endometrialperitoneal interactions during endometriotic lesion establishment. American Journal of Pathology 173 700-715. (doi:10.2353/ajpath. 2008.071128)

Machado DE, Abrão MS, Berardo PT, Takiya CM \& Nasciutti LE: 2008 Vascular density and distribution of vascular endothelial growth factor (VEGF) and its receptor VEGFR-2 (Flk-1) are significantly higher in patients with deeply infiltrating endometriosis affecting the rectum. Fertility and Sterility 90 148-155. (doi:10.1016/j.fertnstert. 2007.05.076)

Machado DE, Berardo PT, Palmero CY \& Nasciutti LE 2010 Higher expression of vascular endothelial growth factor (VEGF) and its receptor VEGFR-2 (Flk-1) and metalloproteinase-9 (MMP-9) in a rat model of peritoneal endometriosis is similar to cancer diseases. Journal of Experimental \& Clinical Cancer Research 29 4. (doi:10.1186/17569966-29-4)

Masuda H, Maruyama T, Hiratsu E, Yamane J, Iwanami A, Nagashima T, Ono M, Miyoshi H, Okano HJ, Ito M et al. 2007 Noninvasive and realtime assessment of reconstructed functional human endometrium in NOD/SCID/gamma c (null) immunodeficient mice. PNAS 104 1925-1930. (doi:10.1073/pnas.0604310104)

McKenna F, McLaughlin PJ, Lewis BJ, Sibbring GC, Cummerson JA, BowenJones D \& Moots RJ 2002 Dopamine receptor expression on human Tand B-lymphocytes, monocytes, neutrophils, eosinophils and NK cells: a flow cytometric study. Journal of Neuroimmunology 132 34-40. (doi:10. 1016/S0165-5728(02)00280-1)

McLaren J, Prentice A, Charnock-Jones DS, Millican SA, Müller KH, Sharkey AM \& Smith SK 1996 Vascular endothelial growth factor is produced by peritoneal fluid macrophages in endometriosis and is regulated by ovarian steroids. Journal of Clinical Investigation 98 482-489. (doi:10.1172/JCI118815)

Meduri G, Bausero P \& Perrot-Applanat M 2000 Expression of vascular endothelial growth factor receptors in the human endometrium: modulation during the menstrual cycle. Biology of Reproduction $\mathbf{6 2}$ 439-447. (doi:10.1095/biolreprod62.2.439)
Moody DM, Thore CR, Anstrom JA, Challa VR, Langefeld CD \& Brown WR 2004 Quantification of afferent vessels shows reduced brain vascular density in subjects with leukoaraiosis. Radiology 233 883-890. (doi:10. 1148/radiol.2333020981)

Nap AW, Griffioen AW, Dunselman GA, Bouma-TerSteege JC, Thijssen VL, Evers JL \& Groothuis PG 2004 Antiangiogenesis therapy for endometriosis. Journal of Clinical Endocrinology and Metabolism 89 1089-1095. (doi:10.1210/jc.2003-031406)

Nisolle M, Casanas-Roux F, Anaf V, Mine JM \& Donnez J 1993 Morphometric study of the stromal vascularization in peritoneal endometriosis. Fertility and Sterility 59 681-684.

Novella-Maestre E, Carda C, Noguera I, Ruiz-Saurí A, Garcia-Velasco JA, Simon C \& Pellicer A 2009 Dopamine agonist administration causes a reduction in endometrial implants through modulation of angiogenesis in experimentally induced endometriosis. Human Reproduction $\mathbf{2 4}$ 1025-1035. (doi:10.1093/humrep/den499)

Pauli SA, Tang H, Wang J, Bohlen P, Posser R, Hartman T, Sauer MV, Kitajewski J \& Zimmermann RC 2005 The vascular endothelial growth factor (VEGF)/VEGF receptor 2 pathway is critical for blood vessel survival in corpora lutea of pregnancy in the rodent. Endocrinology 146 1301-1311. (doi:10.1210/en.2004-0765)

Punyadeera C, Thijssen VL, Tchaikovski S, Kamps R, Delvoux B, Dunselman GA, de Goeij AF, Griffioen AW \& Groothuis PG 2006 Expression and regulation of vascular endothelial growth factor ligands and receptors during menstruation and post-menstrual repair of human endometrium. Molecular Human Reproduction 12 367-375. (doi:10. 1093/molehr/gal027)

Reese J, Binart N, Brown N, Ma WG, Paria BC, Das SK, Kelly PA \& Dey SK 2000 Implantation and decidualization defects in prolactin receptor (PRLR)-deficient mice are mediated by ovarian but not uterine PRLR. Endocrinology 141 1872-1881. (doi:10.1210/en.141.5.1872)

Ricci E, Parazzini F, Motta T, Ferrari Cl, Colao A, Clavenna A, Rocchi F, Gangi E, Paracchi S, Gasperi M et al. 2002 Pregnancy outcome after cabergoline treatment in early weeks of gestation. Reproductive Toxicology 16 791-793. (doi:10.1016/S0890-6238(02)00055-2)

Robert E, Musatti L, Piscitelli G \& Ferrari CI 1996 Pregnancy outcome after treatment with the ergot derivative, cabergoline. Reproductive Toxicology 10 333-337. (doi:10.1016/0890-6238(96)00063-9)

Rockwell LC, Pillai S, Olson CE \& Koos RD 2002 Inhibition of vascular endothelial growth factor/vascular permeability factor action blocks estrogen induced uterine edema and implantation in rodents. Biology of Reproduction 67 1804-1810. (doi:10.1095/biolreprod.102.006700)

Roth BL 2007 Drugs and valvular heart disease. New England Journal of Medicine 356 6-7. (doi:10.1056/NEJMp068265)

Schade R, Andershon S, Suissa S, Haverkamp W \& Garbe E 2007 Dopamine agonists and the risk of cardiac-valve regurgitation. New England Journal of Medicine 356 29-38. (doi:10.1056/NEJMoa062222)

Shifren JL, Tseng JF, Zaloudek CJ, Ryan IP, Meng YG, Ferrara N, Jaffe RB \& Taylor RN 1996 Ovarian steroid regulation of vascular endothelial growth factor in the human endometrium: implications for angiogenesis during the menstrual cycle and in the pathogenesis of endometriosis. Journal of Clinical Endocrinology and Metabolism 81 3112-3118. (doi:10.1210/jc.81.8.3112)

Verhelst J, Abs R, Maiter D, Van den Bruel A, Vandeweghe M, Velkeniers B, Mockel J, Lamberigts G, Petrossians P, Coremans P et al. 1999 Cabergoline in the treatment of hyperprolactinemia: a study of 455 patients. Journal of Clinical Endocrinology and Metabolism 84 2518-2522. (doi:10.1210/jc.84.7.2518)

Walch K, Unfried G, Huber J, Kurz C, van Trotsenburg M \& Pernicka E 2009 Implanon versus medroxyprogesterone acetate: effects on pain scores in patients with symptomatic endometriosis - a pilot study. Contraception 79 29-34. (doi:10.1016/j.contraception.2008.07.017)

Wang D, Stockard CR, Harkins L, Lott P, Salih C, Yuan K, Buchsbaum D, Hashim A, Zayzafoon M, Hardy RW et al. 2008 Immunohistochemistry in the evaluation of neovascularization in tumor xenografts. Biotechnic \& Histochemistry 83 179-189. (doi:10.1080/10520290802451085)

Wulff C, Wilson H, Rudge JS, Wiegand SJ, Lunn SF \& Fraser HM 2001 Luteal angiogenesis: prevention and intervention by treatment with vascular endothelial growth factor trap (A40). Journal of Clinical Endocrinology and Metabolism 86 3377-3386. (doi:10.1210/jc.86.7.3377) 
Zanettini R, Antonini A, Gatto G, Gentile R, Tesei S \& Pezzoli G 2007 Valvular heart disease and the use of dopamine agonists for Parkinson's disease. New England Journal of Medicine 356 39-46. (doi:10.1056/ NEJMoa054830)

Zimmermann RC, Hartman T, Bohlen P, Sauer MV \& Kitajewski J 2001a Preovulatory treatment of mice with anti-VEGF receptor 2 antibody inhibits angiogenesis in corpora lutea. Microvascular Research 62 15-25. (doi:10.1006/mvre.2001.2312)

Zimmermann RC, Xiao E, Husami N, Sauer MV, Lobo R, Kitajewski J \& Ferin M 2001b Short-term administration of antivascular endothelial growth factor antibody in the late follicular phase delays follicular development in the rhesus monkey. Journal of Clinical Endocrinology and Metabolism 86 768-772. (doi:10.1210/jc.86.2.768)
Zimmermann RC, Hartman T, Kavic S, Pauli SA, Bohlen P, Sauer MV \& Kitajewski J 2003 Vascular endothelial growth factor receptor 2-mediated angiogenesis is essential for gonadotropin-dependent follicle development. Journal of Clinical Investigation 112 659-669. (doi:10. 1172/JCI18740)

Received 22 June 2011

First decision 27 July 2011

Revised manuscript received 3 August 2011

Accepted 23 August 2011 